\title{
Transition of the annual cycle of precipitation from double-peak mode to single-peak mode in South China
}

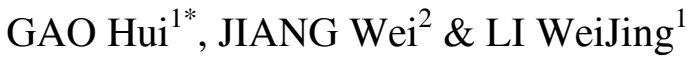 \\ ${ }^{1}$ National Climate Center, China Meteorological Administration, Beijing 100081, China; \\ ${ }^{2}$ Jiangsu Climate Center, Jiangsu Meteorological Bureau, Nanjing 210000, China
}

Received August 28, 2012; accepted December 5, 2012; published online June 6, 2013

\begin{abstract}
Previous studies revealed the double-peak mode (DPM) in South China precipitation, corresponding to the two stages in the rainy season, i.e. the first rainy stage (FRS) and the second rainy stage (SRS). But observations in recent two decades show that the DPM has changed to a single-peak mode (SPM). Both the precipitation amount and the heavy rainfall event frequency enhanced significantly in the gap between the FRS and the SRS in 1991-2010, compared to those in 1961-1990. This change can be linked to the effects of the global warming. During the warmer period, the July sea surface temperature over the western Pacific has greater increases than that over the central and eastern Pacific, especially west of $140^{\circ} \mathrm{E}$. It may generate more tropical cyclones (TCs) in the inshore areas and then more typhoon rainfall over South China. On the other hand, the increments of the air temperature over the East Asian continent are greater than those of the SST over the western Pacific under the global warming, which enlarges the land-ocean temperature/pressure contrast and leads to a trend of the earlier onset dates of the Asian summer monsoon (ASM) in recent two decades. Then, the earlier ASM will facilitate the western Pacific subtropical high (WPSH) to retreat earlier from the South China Sea and enhance the convective precipitation in South China between the FRS and the SRS. Also, due to the warmer ocean, the WPSH locates more westward in July, and more moisture will be transported to South China from the southwest side to the WPSTH. All these influences favor a remarkably increasing precipitation in the gap in the warmer period and changes the seasonal cycle from double-peak mode to single-peak mode.
\end{abstract}

first rainy stage in South China, second rainy stage in South China, double-peak mode, single-peak mode, summer monsoon

Citation: Gao H, Jiang W, Li W J. Transition of the annual cycle of precipitation from double-peak mode to single-peak mode in South China. Chin Sci Bull, 2013, 58: 3994-3999, doi: 10.1007/s11434-013-5905-0

The precipitation over China has significant regional differences and remarkable temporal variations. South China, influenced by both the monsoon system and the tropical cyclone (TC), has the greatest annual rainfall amounts, the most frequent heavy rainfall events, and the longest rainy season in the country. South China covers a coastal area of southern Fujian, Guangdong, Guangxi, Hainan and Taiwan. Distinct from the other regions in China, the precipitation in South China shows a unique double-peak annual cycle [1]. The first rainy stage (FRS) occurs in April-June with the peak in May-June. It is the main rainy period in South China. The total precipitation amount in this stage is about $600-800 \mathrm{~mm}$, accounting for about $40 \%-50 \%$ of the annual

*Corresponding author (email: gaohui@cma.gov.cn) accumulation. Previous studies have shown that the rainfall in the FRS period is mainly composed of the frontal rainfall before the onset of the Asian summer monsoon (ASM) and the summer monsoon rainfall after that [2]. It is found that the frontal rainfall has a close relationship with the anomalous general circulation pattern over the northern Pacific, relating tightly to the north polar vortex and the westerly jet north of the Asian continent [3]. This pattern differs from both the typical summer monsoon circulation and the winter monsoon circulation. That means a complexity in the impact factors of the FRS rainfall. In this stage, the moisture transportation from the western Pacific plays a more important role than that from the Arab Sea and the Bay of Bengal [4]. Studies also explored the consistent interannual variations of the precipitation in FRS, the northeastern cold 
vortex intensity and the Northern Hemisphere annular mode index in late winter and early spring [5,6]. The rainfall in the second rainy stage (SRS) in South China occurs mainly in July-September, i.e. the most active period of both the summer monsoon and tropical cyclones. So, the precipitation in this stage has a close relationship with the ENSO events, the sea surface temperature (SST) over the tropical western Pacific, the convection activities over the South China Sea (SCS), and the frequency of the tropical cyclones $[7,8]$. Besides these impact factors, the sea ice and the general circulation in the Southern Hemisphere, such as the Australian high and the Mascarene high, are also thought to have great impacts on the South China precipitation $[9,10]$. From middle June to early July, affected by the northward movement of the Asian summer monsoon and the western Pacific subtropical high (WPSTH), the main rain belt in China also moves northward to the Yangtze River valley, and the rainfall in South China in this period becomes weak to form a gap between the FRS and the SRS.

In recent two decades, both the positions of the summer rain belt and the beginning/ending dates of the flood season in Chinese experience remarkable changes [11-14]. Studies also indicated that the Chinese Meiyu in the 2000s locates more northward to cover the Huaihe River valley instead of the traditional Yangtze River valley $[12,13]$. For the climate in South China, owing to the complex impact factors, its drought/flood events sometimes alter abruptly [15]. That is to say, the precipitation in this region has strong subseasonal variations and it will bring great challenges to the climate prediction. Most previous studies focused on the traditional FRS and SRS periods only and few of them interested in the rainfall gap between them. But statistical results indicate that the maximal interannual variability of precipitation in South China appears in the gap instead of in the FRS or the SRS period, i.e. in the 30th to the 35 th pentads (about late June to July). In fact, the maximal increases of heavy rainfall event frequency in 1991-2010 occur also in the gap, compared to that in 1961-1990. For example, the heavy flood in July 1994 in South China killed more than 600 people and caused an economic loss of nearly 5 billion dollars. In July 2001, more than 100 people lost their lives due to the persistent typhoon rainfall in Guangdong and Guangxi, and the estimated economic losses were near 2 billion dollars. Therefore, it is necessary to understand the new features of the precipitation in South China and to explore the possible mechanisms by the latest and highresolution observed dataset. The results are expected to provide some useful information for the prevention and reduction of the drought or flood disasters in South China.

\section{Data description}

The observed daily rainfall amounts at 743 Chinese stations are provided by China Meteorological Administration
(CMA). For data quality, only the stations at which the data are completed during 1961-2010 are selected for further study. In this paper, the daily precipitation is accumulated in each 1st-5th, 6th-10th, 11th-15th, 16th-20th, 21st-25th days in every month to get the accumulations of the 1st, 2nd, 3 rd, 4th and 5th pentad, respectively. For the 6th pentad precipitation in each month, the accumulation is the sum from the 26th to the last day.

According to the Chinese geographic and meteorological regionalization [16], the precipitation in South China is defined as the average of the 51 stations in the region south of $25^{\circ} \mathrm{N}$ and east of $105^{\circ} \mathrm{E}$. The tropical cyclone data in northern Pacific are provided by Shanghai Typhoon Institute of CMA. In the data, the aircraft sounding results, the historical documents and the station observations are integrated together [17]. Time series of the South China Sea summer monsoon (SCSSM) onset date in the same period comes from the National Climate Center (NCC) of CMA. The atmospheric datasets used in this paper are obtained from the NCEP/NCAR reanalysis project including the 500-hPa geopotential height, the $850-\mathrm{hPa}$ horizontal wind and the specific humidity on a $2.5^{\circ} \times 2.5^{\circ}$ latitude-longitude grid $[18,19]$. The extended reconstructed sea surface temperature (ERSST) data are provided by NOAA [20].

\section{Transition of the annual cycle of precipitation from double-peak mode to single-peak mode}

The climate in China has clear decadal variations. For example, since the 1990s the air temperatures in both the whole country and South China show remarkable warming trends [21,22]. But the trends are not consistent in different regions and seasons [21,23]. Besides the air temperatures, the precipitation in South China also shows a decadal change since the 1990s. The pentad rainfall amounts in 1961-1990 clearly showed two high value centers during the annual cycle (the center is defined as the pentad precipitation greater than $50 \mathrm{~mm}$, figure not shown). While in 1991-2010, the first center delays and the second center advances so that the two centers merge into one. To better understand the different annual cycles in the two periods, the regional averaged precipitation in South China in 1961-1990 and 1991-2010 are shown in Figure 1. In the first period (thick dashed curve in Figure 1), the precipitation shows a clear double-peak annual cycle. From the 19th pentad (about early April) the precipitation begins to exceed $20 \mathrm{~mm}$ and keeps increasing since then. With the onset of SCSSM, from the 29th pentad the precipitation begins to exceed $40 \mathrm{~mm}$. However, due to the northward jump of both the WPSTH and the summer monsoon, from the 31 st pentad the precipitation begins to decrease. In the 38th pentad, the precipitation reaches its minimum with a value of less than $20 \mathrm{~mm}$. This decrease indicates the ending of the first rainy stage in South China. After a short decrease, the precipitation 


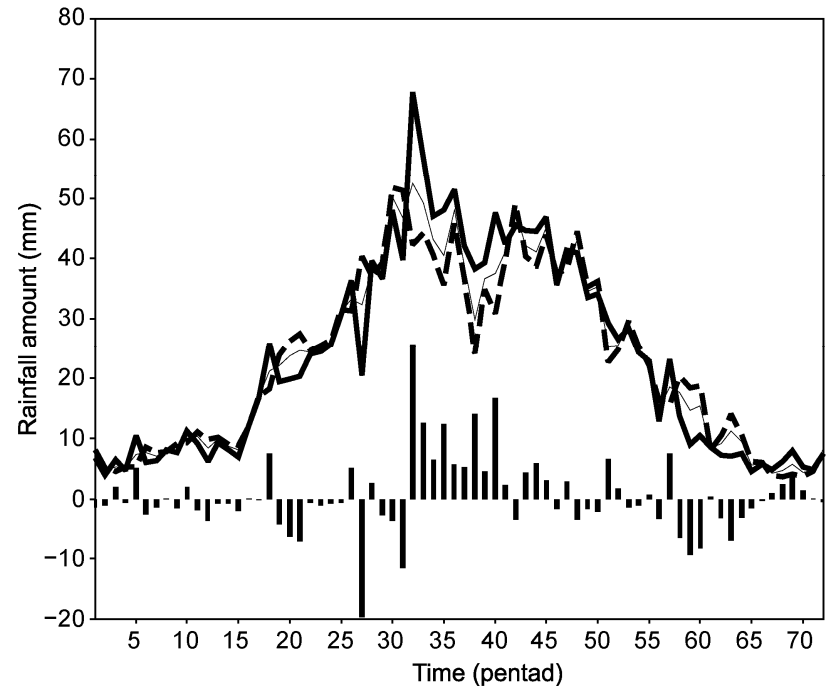

Figure 1 Regional averaged precipitation in South China in each pentad in 1961-1990 (thick dashed curve), 1991-2010 (thick solid curve) and in the whole research period (thin solid curve), respectively. The histogram in the figure means the differences of the precipitation between 1991-2010 and 1961-1990.

begins to increase again and exceeds $40 \mathrm{~mm}$ in the $42 \mathrm{nd}$ pentad (about late July), which means the beginning of the second rainy stage. From Figure 1 it can also be found that the precipitation in July is relatively less compared to that in other main rainy months.

But in the second period, the annual cycle shows an obvious single-peak mode. The maximal value center delays to the 32nd pentad, and the pentad precipitation amount approaches $70 \mathrm{~mm}$. Compared to the precipitation in 19611990 , in this period it increases from middle June to early July, while decreases from middle May to early June. Distinct from the FRS stage, the precipitation in AugustSeptember is almost the same in the two periods, and the difference between them is only about $\pm 5 \mathrm{~mm}$. So it can be said that the remarkable increase in middle June to July in the second period fills the less rainfall gap between the FRS and the SRS and alters the double-peak mode of annual cycle to a single-peak mode.

Linear trend analysis of the pentad precipitation in the whole period also indicates that the significant increasing trends (exceeding the $95 \%$ confidence level of $t$-test) occur only in the $32 \mathrm{nd}$, the 38 th and the 40th pentads. Results also indicate the extreme heavy rainfall events in China increased under the global warming, especially along the coastal regions. Analyses in this paper show the heavy rainfall cases (daily rainfall amount great than $50 \mathrm{~mm}$ ) increased significantly in June and July. In July, the increment is about $30 \%$. While in April-May, the case numbers decreased. And there is no clear trend of heavy rainfall cases in August or September. While to the weak rainfall events (daily rainfall less than $10 \mathrm{~mm}$ ), the trend is not clear in neither of months from March to August. So it can be said that the weakening mode of the double-peak cycle in South China can be attributed to the increased heavy rainfall events in June-July.

\section{Possible mechanisms}

As shown above, the biggest difference of precipitation between the two periods appears in July, so in the following paper we focus mainly on this month. In July, the tropical cyclones become more active and the climatological frequencies over the western Pacific and the South China Sea (SCS) are twice as the numbers in June. So it is necessary to analyze the influences of tropical cyclones to the rainfall in South China. Figure 2 shows the differences of the SSTs in July between the two periods, along with the TC generating places in the same month. Obviously, the SSTs in the second period are warmer in almost all the sea areas, especially

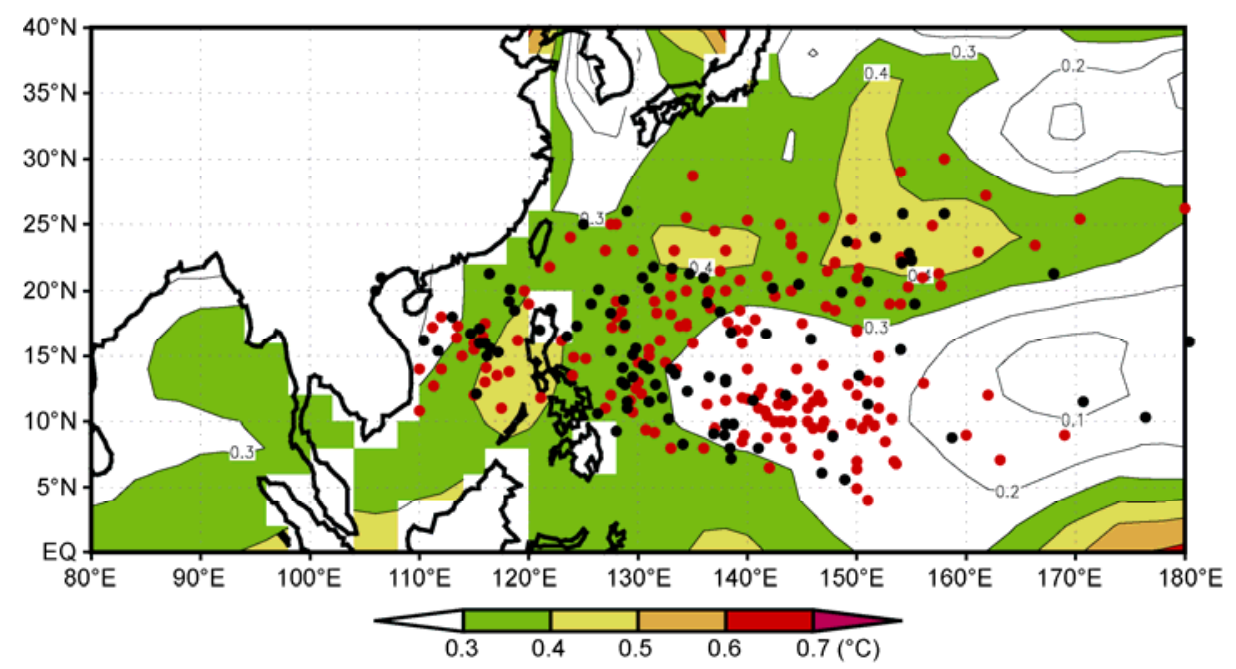

Figure 2 Differences of the SSTs in July between 1991-2010 and 1961-1990. Shaded are values greater than $0.3^{\circ} \mathrm{C}$. The black and red dots mean the generating places of the TCs in 1991-2010 and 1961-1990, respectively. 
in the regions west of $140^{\circ} \mathrm{E}$. This will benefit a more westward location of the generating place of TC. As shown in Figure 2, in recent two decades most TCs were originated in the west part of the western Pacific (black dots), which is quite different from the generating places in the first period (red dots). In 1991-2010, the ratio of the TC numbers originated over the west and east areas of $140^{\circ} \mathrm{E}$ in July increased to $2.4: 1$, compared to $0.99: 1$ in 1961-1990. Undoubtedly, more westward originated TCs will bring more direct influences on the precipitation in South China.

In recent two decades, the air temperatures over the Eurasian continent increase more significantly than the SSTs, and it has advanced the transition of the seasonal land-sea thermal contrast. According to the studies of Zeng and Li [25], the semi-permanent planetary waves due to the seasonal variation of the thermal differences between the ocean and the continent are the secondary forcing of monsoon. Qi et al. [26] also suggested that the seasonal cycle caused by the zonal thermal contrast between the Asian continent and the western Pacific may be an independent driving force for East Asian subtropical monsoon. The precipitation in South China can be affected by both the tropical cyclones and the monsoon systems, especially by the beginning dates of the Asian summer monsoon. So it is supposed that the changed land-sea thermal contrast transition time in recent two decades will modify the onset dates of the ASM and then affect the precipitation. To illustrate it, Figure 3 shows the daily air temperature differences at $850-\mathrm{hPa}$ level between the East Asian continent $\left(20^{\circ}-40^{\circ} \mathrm{N}, 110^{\circ}-120^{\circ} \mathrm{E}\right)$ and the western Pacific $\left(20^{\circ}-40^{\circ} \mathrm{N}, 130^{\circ}-140^{\circ} \mathrm{E}\right)$. Obviously, the transition time in 1961-1990 is later than the date in 1991-2010. In the latter period the transition occurs in early February, while in the former period the date delays to late February. The advanced transition date leads to an earlier onset of the ASM. According to the monitored results of the SCSSM in NCC, the mean onset date in 1991-2010 is one pentad earlier than the time in 1961-1990 (figure not shown).

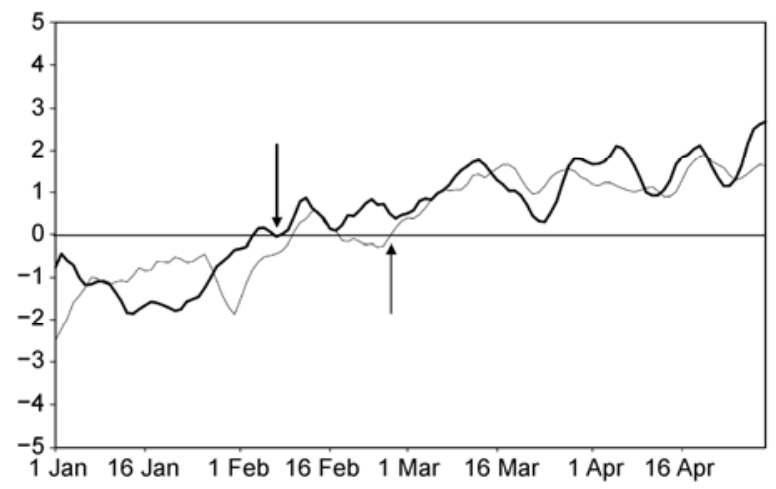

Figure 3 Daily air temperature differences at $850-\mathrm{hPa}$ level between the East Asian continent $\left(20^{\circ}-40^{\circ} \mathrm{N}, 110^{\circ}-120^{\circ} \mathrm{E}\right)$ and the western Pacific $\left(20^{\circ}-40^{\circ} \mathrm{N}, 130^{\circ}-140^{\circ} \mathrm{E}\right)$ in $1991-2010$ (thick curve) and $1961-1990$ (thin curve). The arrows mean the transition time in each period respectively.
After the onset of the ASM, the WPSTH usually moves to the western Pacific from the South China Sea, and the convective precipitation in SCS and South China will be strengthened quickly. So the precipitation in South China has a close relationship with the summer monsoon intensity, especially on their interannual timescales. But the relation is not stable. Results indicated that the quasi-biennial components of the precipitation and the SCS summer monsoon intensity show a weak negative relationship before 1976 while a strong positive relationship since then [27]. Previous researches also suggested the earlier SCSSM will lead to more precipitation in South China. But the relationship also experienced a decadal variation, especially in July. As shown in Figure 4, the relationship between the precipitation and the onset dates of SCSSM is not stable before the middle 1980s. The positive coefficients at 36th and 40th pentad can exceed the $95 \%$ confidence level. But after that, the relationship changed to negative. During the 37th-40th pentad, the negative correlation coefficients can also exceed the same level. So under the global warming, the temperature increments over the land are greater than those over the ocean. This has led to an earlier seasonal transition of the monsoon and brought more monsoon rainfall in South China in July. This is another reason for the weakening doublepeak cycle mode in recent two decades.

The altered relationship can be explained by the changes of the WPSTH and the related moisture transportation (figures not shown). In July, the cross-equatorial flows (CEFs) have been established. To China, the strongest moisture transportation is by the southwesterly from the Indian Ocean and the Bay of Bengal caused by the Somalia CEF. The second transportation, which is from the west side of the WPSTH, is relatively weaker. But to the summer rainfall in South China, the second transportation is considered to be more important [28]. From the analyses mentioned before, the increases of the SSTs in the western Pacific is greater than the counterparts in other Pacific areas, which will expand the air mass and make the WPSTH stronger and more westward (figures not shown). Thus the water vapor in the southwest part of the WPSTH will be carried more directly to South China and will reinforce the vapor convergence at lower troposphere in the region. Influenced by all these factors, the precipitation in the gap between the FRS and the SRS increases sharply and leads to the transition of the annual cycle from DPM to SPM.

\section{Conclusions and discussions}

The flood season in South China is used to be divided into two stages, i.e. the first rainy stage and the second rainy stage. Its annual cycle shows a double-peak mode. Compared to May-June and August-September, July has relatively less precipitation. But in recent two decades, the position, the intensity and the beginning/ending dates of the 


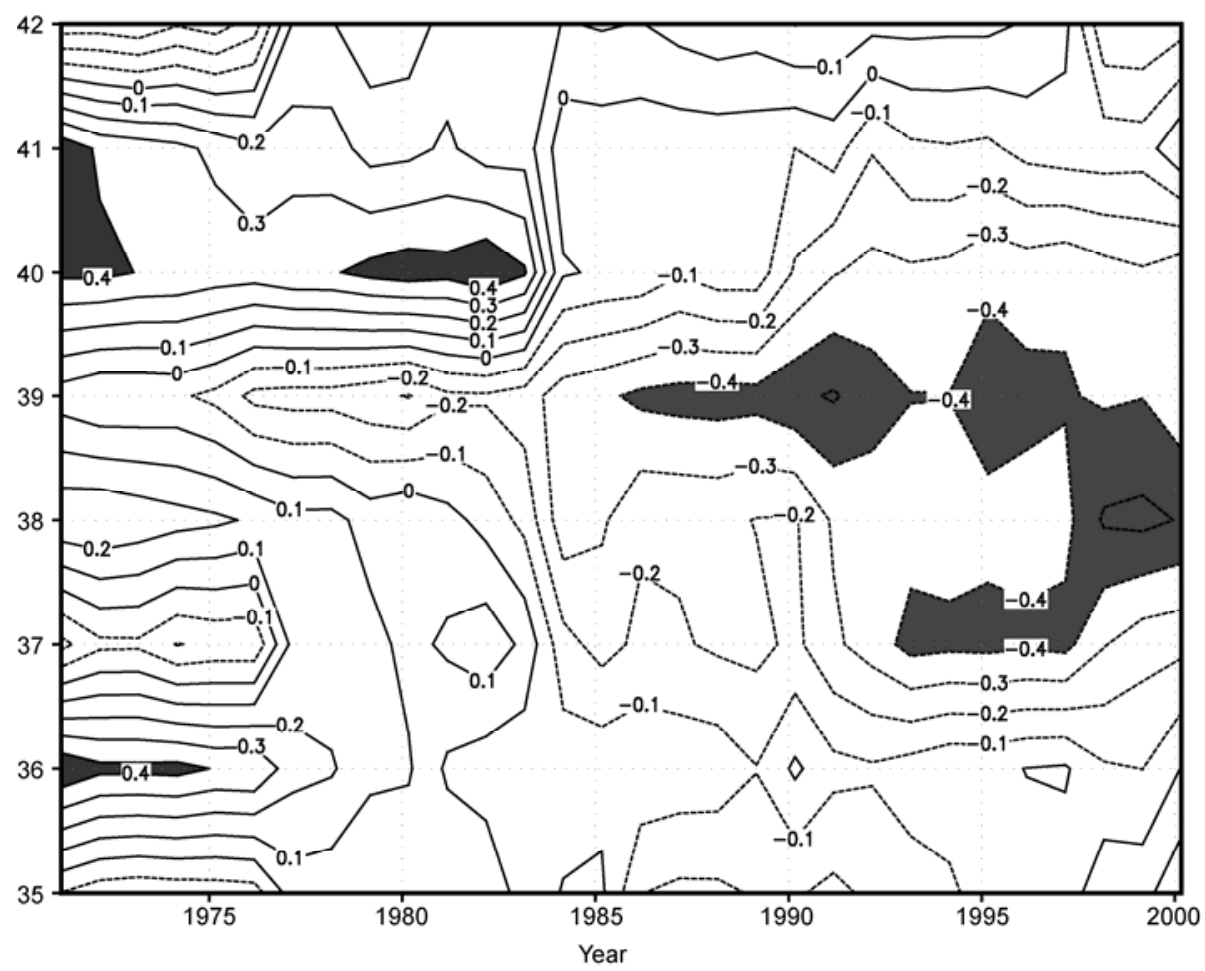

Figure 4 The 21-year running correlation coefficients between the SCSSM onset dates and the precipitation in South China. The number in the abscissa means the central year of the running 21 years (for example, 1981 means the running years are 1971-1991). The number in the ordinate means the pentad. Shaded area means the $95 \%$ confidence level.

summer rainfall in China appear new characteristics. Particular, in South China, the precipitation shows a single-peak cycle mode instead of the traditional double-peak cycle. The precipitation, especially the heavy rainfall, has increased significantly to fill the less rainfall gap between the FRS and the SRS. Results indicate that both the maximal variation and the biggest increment occur in July rather than in other months.

Composed results indicate the SSTs over the tropical western Pacific have increased since the 1990s, especially over the regions west of $140^{\circ} \mathrm{E}$. The warmer SSTs over the western Pacific will make tropical cyclones generated more westward. In recent two decades, the ratio of the TC numbers originated over the west and east regions of $140^{\circ} \mathrm{E}$ in Pacific in July is about $2.4: 1$, while the value is $0.99: 1$ in 1961-1990. Under this pattern, the typhoon rainfall becomes more in South China.

In 1991-2010, the temperatures of the Eurasian continent also increase significantly and the increment is greater than that in the ocean. Thus the seasonal transition of the landocean thermal contrast has been enhanced. In this warmer period, the SCSSM appears earlier and the WPSTH moves also earlier to the western Pacific. So the convection over South China is stronger and it brings more monsoon rainfall to this region. On the other hand, owing to the warmer SSTs over the western Pacific, the WPSTH locates more westward in recent two decades. Thus the moisture in the southwest part of the WPSTH will be carried more directly to South China and reinforced the water vapor convergence at lower troposphere. Influenced by all these factors, the precipitation in the gap between the FRS and the SRS increased remarkably and contributes to the transition of the annual cycle from DPM to SPM.

Besides the general circulations and the SSTs, the snow conditions over the Tibetan Plateau are also known to have a close relationship with the precipitation in South China. Results indicated that more summer rainfall will occur in this region after a less snow cover winter, and vice versa. The anomalies of snow cover in previous winter and spring will alter the thermal conditions over the Eurasian continent and then change the general circulations in both South Asia and East Asia [29,30]. This mechanism has also been proved in the numerical simulations [31]. So it could be hypothesized that the changed annual cycle in South China could be partly attributed to the changed snow condition over the Tibetan Plateau. It is quite important to validate it though previous studies focused only on the summer precipitation instead of the other seasons. So in the next step the authors will analyze the possible influences of the snow conditions on the modified annual cycles in South China precipitation.

The authors kindly thank the reviewers for their valuable comments and suggestions that greatly helped to improve the article. This work was supported by the National Basic Research Program of China (2012CB417205 and 2013CB430203), the International Cooperation Program of Ministry of 
Science and Technology of China (2009DFA23010), the Special Research Fund (Meteorology) of Public Welfare Project (GYHY201306033) and the National Natural Science Foundation of China (41005037). The authors also thank the support of the National Innovation Team of Climate Prediction of China Meteorological Administration.

1 Guo Q Y, Sha W Y. Analysis of rainfall variability during the first rainy season in South China (in Chinese). J Appl Meteorol Sci, 1998, 9: 9-15

2 Chi Y Z, He J H, Wu Z W. Features analysis of the different precipitation periods in the pre-flood season in South China (in Chinese). J Nanjing Inst Meteorol, 2005, 28: 163-171

3 Wang H J, Xue F, Zhou G Q. The spring monsoon in South China and its relationship to large-scale circulation features. Adv Atmos Sci, 2002, 19: 651-664

4 Chi Y Z. Features of the precipitation during the pre-flood season in South China and its interaction with South China Sea summer monsoon. Dissertation for the Master Degree (in Chinese). Nanjing: Nanjing University of Information, Science and Technology, 2005. 1-76

5 Miao C S, Wu Z W, He J H, et al. Northeast cold vortex during the first flood period in the last 50 years and its correlation with rainfall in South China (in Chinese). Chin J Geophys, 2006, 30: 1249-1256

6 Miao C S, Wu Z W, He J H. Relationship among the Northern Hemisphere annual mode, the northeast China cold vortex and precipitation during the first yearly rainy period in South China (in Chinese). J Trop Meteorol, 2006, 6: 593-599

7 He Y H, Guan C H, Lin X G, et al. Oscillation and distribution of rainfall during second rainy season in South China (in Chinese). J Trop Meteorol, 1998, 14: 359-383

8 Ren G C, Fan F L, Shen A H. Comparison researches on the rainfall of the former and latter flood season in the southeast coastal area of China (in Chinese). Marine Forecasts, 2007, 24: 16-24

9 Zhang A H, Qin W, Wu H Q, et al. Relationship of the precipitation in the second rainy stage in South China with the Antarctic sea ice and the general circulations in Southern Hemisphere (in Chinese). Guangxi Meteorol, 1998, 19: 2-7

10 Xue F, Wang $\mathrm{H} \mathrm{J}$, He J H. Interannual variability of Mascarene high and Australian high and their influences on summer rainfall over East Asia. Chin Sci Bull, 2003, 48: 492-497

11 Wang $\mathrm{H}$ J. The weakening of the Asian monsoon circulation after the end of 1970s. Adv Atmos Sci, 2001, 18: 376-386

12 Zhao Z G, Zhu Y F, Liu Y X, et al. Decadal variation of summer rain-pattern in China during 1880-2006 (in Chinese). Adv Clim Change Res, 2008, 4: 95-100

13 Si D, Ding Y H, Liu Y J. Decadal northward shift of the Meiyu belt and the possible cause. Chin Sci Bull, 2009, 54: 4742-4748

14 Zhu Y L, Wang H J, Zhou W, et al. Recent changes in the summer precipitation pattern in East China and the background circulation. Clim Dyn, 2010, 10: 852-860
$15 \mathrm{Wu}$ Z W, Li J P, He J H, et al. Statistical analyses of the coexistence and alteration of the flood and drought events in South China in summer. Prog Nat Sci, 2007, 17: 1665-1671

16 Guo J X, Wang X R, Wang W G. Geographic and Meteorological Regionalization of China (in Chinese). Beijing: China Meteorological Press, 2006. 1-72

17 Liang J, Ren F M, Yang X Q. Study on the differences between CMA and JTWC tropical cyclone datasets for northwest Pacific (in Chinese). Acta Oceanol Sin, 2010, 2: 10-22

18 Kalnay E, Kanamitsu M, Kistler R, et al. The NCEP/NCAR 40-year reanalysis project. Bull Am Meteorol Soc, 1996, 77: 437-471

19 Kistler R, Collins W, Saha S, et al. The NCEP-NCAR 50-year reanalysis: monthly means CD-ROM and documentation. Bull Am Meteorol Soc, 2001, 82: 247-268

20 Smith T M, Richard W R. Improved extended reconstruction of SST (1854-1997). J Clim, 2004, 17: 2466-2477

21 Ren G Y, Xu M Z, Chu Z Y, et al. Changes of surface air temperature in China during 1951-2004 (in Chinese). Clim Environ Res, 2005, 10: 717-727

22 Tang G L, Ren G Y. Reanalysis of surface air temperature change of the last 100 years over China (in Chinese). Clim Environ Res, 2005, 10: 791-798

23 Yu H Y, Liu S H, Zhao N, et al. Characteristics of air temperature and precipitation in different regions of China from 1951 to 2009 (in Chinese). J Meteorol Environ, 2011, 27: 1-11

24 Ding Y H, Ren G Y, Lin E D, et al. National Assessment Report of Climate Change (in Chinese). Beijing: Science Press, 2007. 34-35

25 Zeng Q C, Li J P. Interactions between the northern and southern hemispheric atmospheres and the essence of monsoon (in Chinese). Chin J Atmos Sci, 2002, 26: 433-448

26 Qi L, He J H, Zhang Z Q, et al. Seasonal cycle of the zonal land-sea thermal contrast and East Asian subtropical monsoon circulation. Chin Sci Bull, 2008, 53: 131-136

27 Zhen B, Shi N. Effects of South China Sea summer monsoon on the interdecadal variability of summer rainfall over South China (in Chinese). J Nanjing Inst Meteorol, 2006, 29: 477-483

28 Wang H J, Chen H P. Climate control for southeastern China moisture and precipitation: Indian or East Asian monsoon? J Geophys Res, 2012, 117: D12109

29 Wu T W, Qian Z A. Further analyses of the linkage between winter and spring snow depth anomaly over Qinghai-Xizang plateau and summer rainfall of eastern China (in Chinese). Acta Meteorol Sin, 2000, 58: 570-581

30 Zhu Y X, Ding Y H, Xu H G. The decadal relationship between atmospheric heat source of winter and spring snow over Tibetan Plateau and rainfall in East China (in Chinese). Acta Meteorol Sin, 2007, 65: $946-958$

31 Gao R, Zhong H L, Dong W J, et al. Impact of snow cover and frozen soil in the Tibetan Plateau on summer precipitation in China (in Chinese). J Glaciol Geocryol, 2011, 33: 254-260

Open Access This article is distributed under the terms of the Creative Commons Attribution License which permits any use, distribution, and reproduction in any medium, provided the original author(s) and source are credited. 